\title{
An arithmetical equation with respect to regular convolutions
}

\author{
Pentti HaukKanen \\ School of Information Sciences, \\ FI-33014 University of Tampere, Finland \\ e-mail: pentti.haukkanen@uta.fi
}

\begin{abstract}
It is well known that Euler's totient function $\phi$ satisfies the arithmetical equation $\phi(m n) \phi((m, n))=\phi(m) \phi(n)(m, n)$ for all positive integers $m$ and $n$, where $(m, n)$ denotes the greatest common divisor of $m$ and $n$. In this paper we consider this equation in a more general setting by characterizing the arithmetical functions $f$ with $f(1) \neq 0$ which satisfy the arithmetical equation $f(m n) f((m, n))=$ $f(m) f(n) g((m, n))$ for all positive integers $m, n$ with $m, n \in A(m n)$, where $A$ is a regular convolution and $g$ is an $A$-multiplicative function. Euler's totient function $\phi_{A}$ with respect to $A$ is an example satisfying this equation.
\end{abstract}

Mathematics Subject Classification (2010). 11A25

Keywords. Euler's totient function, arithmetical equation, quasimultiplicative function, regular convolution

\section{Introduction}

An arithmetical function $f$ is said to be multiplicative if $f(1)=1$ and $f(m n)=f(m) f(n)$ for all positive integers $m, n$ with $(m, n)=1$, where $(m, n)$ denotes the greatest common divisor of $m$ and $n$. A multiplicative function $f$ is said to be completely multiplicative if $f(m n)=f(m) f(n)$ for all positive integers $m, n$. It is well known that Euler's totient function $\phi$ is 
multiplicative but not completely multiplicative. Thus $\phi$ is in a sense "between completely multiplicative and multiplicative functions". A well-known equation that reflects this property is given as

$$
\phi(m n) \phi((m, n))=\phi(m) \phi(n)(m, n)
$$

for all positive integers $m, n$, see e.g. [2]. For all positive integers $m, n$ with $(m, n)=1$ this reduces to $\phi(m n)=\phi(m) \phi(n)$ showing that $\phi$ is multiplicative. Applying the formula

$$
\phi(n)=n \prod_{p \mid n}\left(1-\frac{1}{p}\right)
$$

we see that $\phi(m n) \neq \phi(m) \phi(n)$ for all positive integers $m, n$ with $(m, n)>1$.

An interesting question is to characterize the arithmetical functions satisfying an identity of the type of (1). We could characterize the arithmetical functions $f$ with $f(1)=1$ satisfying $f(m n) f((m, n))=f(m) f(n)(m, n)$ for all $m, n$. It is however easy to consider a slightly more general problem, namely, to characterize the arithmetical functions $f$ with $f(1)=1$ satisfying

$$
f(m n) f((m, n))=f(m) f(n) g((m, n))
$$

for all positive integers $m, n$, where $g$ is a completely multiplicative function. In fact, Apostol and Zuckerman [3] have shown that an arithmetical function $f$ with $f(1)=1$ satisfies (2) if and only if $f$ is multiplicative and

$$
f\left(p^{a+b}\right) f\left(p^{b}\right)=f\left(p^{a}\right) f\left(p^{b}\right) g\left(p^{b}\right)
$$

for all primes $p$ and integers $a \geq b \geq 1$.

We obtain a more illustrative result if we assume that $f$ possesses Property $O$ which is defined as follows. We say that an arithmetical function $f$ satisfies Property $O$ if for each prime $p, f(p)=0$ implies $f\left(p^{a}\right)=0$ for all $a>1$. Then (2) is a characterization of totients. An arithmetical function $f$ is said to be a totient if there are completely multiplicative functions $f_{T}$ and $f_{V}$ such that

$$
f=f_{T} * f_{V}^{-1},
$$

where $*$ denotes the Dirichlet convolution and $f_{V}^{-1}$ is the inverse of $f_{V}$ under the Dirichlet convolution. The functions $f_{T}$ and $f_{V}$ are referred to as the integral and inverse part of $f$. Now we are in a position to present the promised characterization of totients (given in [8]). An arithmetical function $f$ is a totient if and only if $f$ with $f(1)=1$ satisfies Property $O$ and there 
exists a completely multiplicative function $g$ such that (2) holds. In this case $f_{T}=g$.

It is well known that Euler's totient function $\phi$ can be written as

$$
\phi=N * \mu=N * \zeta^{-1},
$$

where $N(n)=n$ and $\zeta(n)=1$ for all positive integers $n$ and $\mu$ is the Möbius function. Thus $\phi$ is a totient in the sense of (4) with $\phi_{T}=N$ and $\phi_{V}=\zeta$. In this case (2) reduces to (1). Any arithmetical function $f$ with $f(1)=1$ and Property $O$ satisfying $f(m n) f((m, n))=f(m) f(n)(m, n)$ for all positive integers $m$ and $n$ is a totient with $f_{T}=N$, that is, $f=N * f_{V}^{-1}$. Dedekind's totient $\psi$ defined as $\psi=N *|\mu|$ is another totient with this property, since $|\mu|=\lambda^{-1}$, where $|\mu|(n)=|\mu(n)|$ is the absolute value of the the Möbius function and $\lambda$ is Liouville's function, which the completely multiplicative function such that $\lambda(p)=-1$ for all primes $p$. Thus Dedekind's totient $\psi$ satisfies the arithmetical equation

$$
\psi(m n) \psi((m, n))=\psi(m) \psi(n)(m, n)
$$

for all positive integers $m, n$.

In this paper we investigate the arithmetical equation (2) in a more general setting. Namely, we introduce a generalization of (2) for regular convolutions. Suppose that $A$ is a regular convolution and $g$ is an $A$-multiplicative arithmetical function (defined in Section 2). Then we consider the arithmetical functions $f$ with $f(1) \neq 0$ which satisfy the arithmetical equation

$$
f(m n) f((m, n))=f(m) f(n) g((m, n))
$$

for all positive integers $m, n$ with $m, n \in A(m n)$. (Note that the condition $m, n \in A(m n)$ is equivalent to the condition $m \in A(m n)$.)

In the case of the Dirichlet convolution the arithmetical equation (6) becomes (2). Equation (6) has not hitherto been studied in the literature. Equation (2) has been studied in $[1,3,6,8]$. For further material relating to this type of equations we refer to $[4,13]$. Theorems $1,3,4$ of this paper are generalizations of Theorems 2, 3 and 4 of [3]. Theorem 2 generalizes the main theorem of [6], and Corollary 2 generalizes Theorem 3 of [1] (see also Lemma 4.1 of [5]). Corollary 1 generalizes Theorem 10 of [8] and shows that the functional equation (6) is closely related to totient type functions.

\section{Preliminaries}

For each positive integer $n$ let $A(n)$ be a subset of the set of positive divisors of $n$. Then the $A$-convolution [11] of two arithmetical functions $f$ and $g$ is 
defined by

$$
\left(f *_{A} g\right)(n)=\sum_{d \in A(n)} f(d) g(n / d)
$$

An $A$-convolution is said to be regular [11] if

(i) the set of arithmetical functions forms a commutative ring with identity with respect to the usual addition and the $A$-convolution,

(ii) the multiplicativity of $f$ and $g$ implies the multiplicativity of $f *_{A} g$,

(iii) the function $\zeta$ has an inverse $\mu_{A}$ with respect to the $A$-convolution, and $\mu_{A}(n)=0$ or -1 whenever $n(\neq 1)$ is a prime power.

The inverse of an arithmetical function $f$ with $f(1) \neq 0$ with respect to an $A$-convolution satisfying condition (i) is defined by

$$
f^{-1} *_{A} f=f *_{A} f^{-1}=\delta,
$$

where $\delta$ is the arithmetical function such that $\delta(1)=1$ and $\delta(n)=0$ for $n>1$.

It is known [11] that an $A$-convolution is regular if and only if

(a) $A(m n)=\{d e: d \in A(m), d \in A(n)\}$ whenever $(m, n)=1$,

(b) for each prime power $p^{a}$ with $a>0$ there exists a positive integer $t$ $\left(=\tau_{A}\left(p^{a}\right)\right)$ such that

$$
A\left(p^{a}\right)=\left\{1, p^{t}, p^{2 t}, \ldots, p^{s t}\right\}
$$

where $s t=a$ and

$$
A\left(p^{i t}\right)=\left\{1, p^{t}, p^{2 t}, \ldots, p^{i t}\right\}, 0 \leq i \leq s .
$$

For example the Dirichlet convolution $D$, defined by $D(n)=\{d>$ $0: d \mid n\}$, and the unitary convolution $U$, defined by $U(n)=\{d>0$ : $d \mid n,(d, n / d)=1\}$, are regular convolutions. We assume throughout this paper that $A$ is an arbitrary but fixed regular convolution.

A positive integer $n$ is said to be $A$-primitive if $A(n)=\{1, n\}$. For each $A$-primitive prime power $p^{t}(\neq 1)$ the order [7] of $p^{t}$ is defined by

$$
o\left(p^{t}\right)=\sup \left\{s: \tau_{A}\left(p^{s t}\right)=t\right\} .
$$

For the Dirichlet convolution $D$ the primes are the only $D$-primitive prime powers and the order of each prime is infinity. For the unitary convolution 
$U$ all prime powers are $U$-primitive and the order of each prime power $(\neq 1)$ is equal to 1 .

In this paper we often write $1 \leq i \leq o\left(p^{t}\right)$. Here $i$ is an integer, and if $o\left(p^{t}\right)=\infty$, we adopt the convention that $1 \leq i \leq o\left(p^{t}\right)$ means $1 \leq i$ (that is, $i$ is a positive integer).

An arithmetical function $f$ is said to be quasi- $A$-multiplicative [7] if $f(1) \neq 0$ and

$$
f(1) f(m n)=f(m) f(n) \text { whenever } m, n \in A(m n) .
$$

It can be shown that a quasimultiplicative function $f$ is quasi- $A$-multiplicative if and only if

$$
f\left(p^{i t}\right)=f(1)^{1-i} f\left(p^{t}\right)^{i}, \quad 1 \leq i \leq o\left(p^{t}\right),
$$

where $p^{t}(\neq 1)$ is an $A$-primitive prime power. Thus a quasi- $A$-multiplicative function is totally determined by its values at $A$-primitive prime powers.

Quasi- $A$-multiplicative functions $f$ with $f(1)=1$ are said to be $A$ multiplicative functions [15]. An arithmetical function $f$ with $f(1) \neq 0$ is quasi- $A$-multiplicative if and only if $f / f(1)$ is $A$-multiplicative. Quasi$U$-multiplicative functions are known as quasimultiplicative functions [9], $U$-multiplicative functions are the usual multiplicative functions, and $D$ multiplicative functions are the usual completely multiplicative functions. Therefore, for example, quasimultiplicative functions are defined by the conditions

$$
\begin{aligned}
& f(1) \neq 0, \\
& f(1) f(m n)=f(m) f(n) \text { whenever }(m, n)=1 .
\end{aligned}
$$

An arithmetical function $f$ is said to be a quasi- $A$-totient [7] if

$$
f=f_{T} *_{A} f_{V}^{-1}
$$

where $f_{T}$ and $f_{V}$ are quasi- $A$-multiplicative functions. The inverse of a quasi$A$-multiplicative function $g$ is given as

$$
g^{-1}=\frac{\mu_{A} g}{g(1)^{2}}
$$

see [7]; thus a quasi- $A$-totient $f$ can be written in the form

$$
f=f_{T} *_{A}\left(\frac{\mu_{A} f_{V}}{f_{V}(1)^{2}}\right) .
$$


The generalized Möbius function $\mu_{A}$ is the multiplicative given by

$$
\mu_{A}\left(p^{a}\right)= \begin{cases}1 & \text { if } a=0 \\ -1 & \text { if } p^{a}(\neq 1) \text { is } A \text {-primitive } \\ 0 & \text { otherwise }\end{cases}
$$

An arithmetical function $f$ with $f(1) \neq 0$ is a quasi- $A$-totient if and only if $f / f(1)$ is an $A$-totient. It is easy to see that $D$-totients are the usual totients and $U$-totients are simply the usual multiplicative functions.

The function $\phi_{A}(n)$ is defined as the number of integers $a(\bmod n)$ such that $(a, n)_{A}=1$, where $(a, n)_{A}$ is the grestest divisor of $a$ that belongs to $A(n)$. It is well known [10] that $\phi_{A}=N *_{A} \mu_{A}$, and therefore $\phi_{A}$ is an $A$-totient with $f_{T}=N$ and $f_{V}=\zeta$.

Dedekind's totient function $\psi_{A}$ with respect to a regular convolution is defined as $\psi_{A}=N *_{A}\left|\mu_{A}\right|$. Let $\lambda_{A}$ denote Liouville's function with respect to a regular convolution, that is, $\lambda_{A}$ is the $A$-multiplicative function such that $\lambda\left(p^{t}\right)=-1$ for all $A$-primitive prime powers $p^{t}(\neq 1)$. Then $\psi_{A}=N *_{A} \lambda_{A}^{-1}$, and therefore $\psi_{A}$ is the $A$-totient with $f_{T}=N$ and $f_{V}=\lambda_{A}$.

For general accounts on regular convolutions and related arithmetical functions we refer to $[10,12,14]$.

\section{Characterization}

We characterize the arithmetical functions $f$ with $f(1) \neq 0$ satisfying (6). We assume that $g$ is an $A$-multiplicative function in (6). Then $g(1)=1$.

Theorem 1. An arithmetical function $f$ with $f(1) \neq 0$ satisfies (6) if and only if $f$ is quasimultiplicative and

$$
f\left(p^{(a+b) t}\right) f\left(p^{b t}\right)=f\left(p^{a t}\right) f\left(p^{b t}\right) g\left(p^{b t}\right)
$$

for all $A$-primitive prime powers $p^{t}(\neq 1)$ and $a \geq b \geq 1, a+b \leq o\left(p^{t}\right)$.

Proof. Assume that (6) holds. Then taking $(m, n)=1$ in (6) gives $f(m n) f(1)=f(m) f(n)$, and therefore $f$ is quasimultiplicative. Furthermore, taking $m=p^{a t}, n=p^{b t}$ with $a+b \leq o\left(p^{t}\right)$ (where $p^{t}(\neq 1)$ is an $A$-primitive prime power) in (6) proves (8).

Conversely, assume that $f$ is a quasimultiplicative function satisfying (8). We show that (6) holds. Since $f$ is quasimultiplicative, it is enough to show that (6) holds when $m$ and $n$ are prime powers. If $m=1$ or $n=1$, then (6) holds. If $m \neq 1$ and $n \neq 1$, then there is an $A$-primitive prime power $p^{t}(\neq 1)$ 
such that $m=p^{a t}, n=p^{b t}$ with $2 \leq a+b \leq o\left(p^{t}\right)$, since $m, n \in A(m n)$. Then (6) reduces to (8), and therefore, by assumption, (6) holds. This completes the proof.

Making a further assumption on $f$ we see that (6) is closely related to totient type functions.

Property $O_{A}$. We say that an arithmetical function $f$ satisfies Property $O_{A}$ if for each $A$-primitive prime power $p^{t}(\neq 1), f\left(p^{t}\right)=0$ implies $f\left(p^{a t}\right)=0$ for all $1 \leq a \leq o\left(p^{t}\right)$.

By virtue of (7), all quasi- $A$-multiplicative functions possess Property $O_{A}$. Since $\phi_{A}\left(p^{t}\right)=p^{t}-1 \neq 0$ and $\psi_{A}\left(p^{t}\right)=p^{t}+1 \neq 0$, the functions $\phi_{A}$ and $\psi_{A}$ possess Property $O_{A}$.

Theorem 2. An arithmetical function $f$ with $f(1) \neq 0$ is a solution of (6) with Property $O_{A}$ if and only if $f$ is quasimultiplicative and there is an $A$ multiplicative function $g$ such that

$$
f\left(p^{a t}\right)=f\left(p^{t}\right) g\left(p^{t}\right)^{a-1}
$$

for all A-primitive powers $p^{t}$ and $1 \leq a \leq o\left(p^{t}\right)$.

Proof. Assume that (6) and Property $O_{A}$ hold. Then (8) holds by Theorem 2. Taking $b=1$ in (8) we obtain

$$
f\left(p^{(a+1) t}\right) f\left(p^{t}\right)=f\left(p^{a t}\right) f\left(p^{t}\right) g\left(p^{t}\right),
$$

where $2 \leq a+1 \leq o\left(p^{t}\right)$. In this way we see that

$$
\begin{aligned}
f\left(p^{(a+1) t}\right) f\left(p^{t}\right) & =f\left(p^{a t}\right) f\left(p^{t}\right) g\left(p^{t}\right)=f\left(p^{(a-1) t}\right) f\left(p^{t}\right) g\left(p^{t}\right)^{2}=\cdots \\
& =f\left(p^{t}\right) f\left(p^{t}\right) g\left(p^{t}\right)^{a},
\end{aligned}
$$

where $2 \leq a+1 \leq o\left(p^{t}\right)$. Thus

$$
f\left(p^{a t}\right) f\left(p^{t}\right)=f\left(p^{t}\right) f\left(p^{t}\right) g\left(p^{t}\right)^{a-1},
$$

where $2 \leq a \leq o\left(p^{t}\right)$. Clearly (10) holds even for $1 \leq a \leq o\left(p^{t}\right)$. If $f\left(p^{t}\right) \neq 0$, then (10) reduces to (9). If $f\left(p^{t}\right)=0$, then (9) holds by Property $O_{A}$.

Conversely, assume that $f$ is a quasimultiplicative function satisfying (9). Let $a \geq b \geq 1, a+b \leq o\left(p^{t}\right)$. Applying (9) we obtain

$$
\begin{aligned}
f\left(p^{(a+b) t}\right) f\left(p^{b t}\right) & =f\left(p^{t}\right) g\left(p^{t}\right)^{a+b-1} f\left(p^{t}\right) g\left(p^{t}\right)^{b-1} \\
& =f\left(p^{t}\right) g\left(p^{t}\right)^{a-1} f\left(p^{t}\right) g\left(p^{t}\right)^{b-1} g\left(p^{b t}\right) \\
& =f\left(p^{a t}\right) f\left(p^{b t}\right) g\left(p^{b t}\right),
\end{aligned}
$$

which shows that (8) holds. Thus, by Theorem $1, f$ is a solution of (6). On the basis of $(9)$ we see that Property $O_{A}$ holds. This completes the proof. 
Lemma 1 ([7]). Suppose that $f$ is a quasi-A-totient. Then

$$
f\left(p^{a t}\right)=\left(\frac{f_{T}\left(p^{t}\right)}{f_{T}(1)}\right)^{a-1} f\left(p^{t}\right), 1 \leq a \leq o\left(p^{t}\right),
$$

for all A-primitive prime powers $p^{t}(\neq 1)$.

Conversely, suppose that $f$ is quasimultiplicative and for all A-primitive prime powers $p^{t}(\neq 1)$ there exists a complex number $z\left(p^{t}\right)$ such that

$$
f\left(p^{a t}\right)=\left(z\left(p^{t}\right)\right)^{a-1} f\left(p^{t}\right), 1 \leq a \leq o\left(p^{t}\right) .
$$

Then $f$ is a quasi-A-totient with $f_{T}\left(p^{t}\right) / f_{T}(1)=z\left(p^{t}\right)$.

Corollary 1. Suppose that $f$ is a quasi-A-totient. Then

$$
f(m n) f((m, n)) f_{T}(1)=f(m) f(n) f_{T}((m, n))
$$

whenever $m, n \in A(m n)$.

Conversely, suppose that there exists a quasi-A-multiplicative function $g$ such that

$$
f(m n) f((m, n)) g(1)=f(m) f(n) g((m, n))
$$

whenever $m, n \in A(m n)$, and $f$ satisfies Property $O_{A}$. Then $f$ is a quasi-Atotient with

$$
\frac{f_{T}\left(p^{t}\right)}{f_{T}(1)}=\frac{g\left(p^{t}\right)}{g(1)} .
$$

Proof. Suppose that $f$ is a quasi- $A$-totient. Then $f$ is quasimultiplicative. By Lemma $1, f$ satisfies (9) with $g\left(p^{t}\right)=f_{T}\left(p^{t}\right) / f_{T}(1)$. Thus, by Theorem 2 , $f$ satisfies (6) with $g=f_{T} / f_{T}(1)$. This means that (11) holds.

Conversely, suppose that $f$ satisfies (12) and Property $O_{A}$. Then $f$ satisfies (6) with $g$ replaced with $g / g(1)$, which is $A$-multiplicative. Thus, by Theorem 2 and Lemma 1, is a quasi- $A$-totient with $f_{T}\left(p^{t}\right) / f_{T}(1)=g\left(p^{t}\right) / g(1)$.

Example 1. Euler's totient function $\phi_{A}$ with respect to a regular convolution satisfies the arithmetical equation

$$
\phi_{A}(m n) \phi_{A}((m, n))=\phi_{A}(m) \phi_{A}(n)(m, n)
$$

whenever $m, n \in A(m n)$, and Dedekind's totient function $\psi_{A}$ with respect to a regular convolution satisfies the same arithmetical equation

$$
\psi_{A}(m n) \psi_{A}((m, n))=\psi_{A}(m) \psi_{A}(n)(m, n)
$$

whenever $m, n \in A(m n)$. 
Corollary 2. Suppose that $g$ is a quasi-A-multiplicative function and $h$ is a multiplicative function with

$$
g\left(p^{t}\right)\left[g\left(p^{t}\right)-h\left(p^{t}\right)\right] \neq 0
$$

for all $A$-primitive prime powers $p^{t}(\neq 1)$. Denote $f=g *_{A}\left(\mu_{A} h\right)$. Then

$$
f(m n)=f(m) f(n) \frac{g((m, n))}{f((m, n)) g(1)}
$$

whenever $m, n \in A(m n)$.

Proof. Let $u$ be the $A$-multiplicative function such that $u\left(p^{t}\right)=h\left(p^{t}\right)$ for all $A$-primitive prime powers $p^{t}(\neq 1)$. Then $u^{-1}=\mu_{A} u=\mu_{A} h$, and thus $f=g *_{A} u^{-1}$, which means that $f$ is a quasi- $A$-totient with $f_{T}=g$. This implies that $f(1) \neq 0$. Further, since $f\left(p^{a t}\right)=g\left(p^{t}\right)^{a-1}\left[g\left(p^{t}\right)-h\left(p^{t}\right)\right] \neq 0$ for all $A$-primitive prime powers $p^{t}(\neq 1)$ and $1 \leq a \leq o\left(p^{t}\right)$ and since $f$ is multiplicative, $f$ is always nonzero. Now, the claim follows from Corollary 1 .

Next we examine (8) without assuming Property $O_{A}$. We distinguish two cases: $g\left(p^{t}\right)=0, g\left(p^{t}\right) \neq 0$.

Theorem 3. Let $p^{t}(\neq 1)$ be an A-primitive prime power, and let $g$ be an A-multiplicative function with $g\left(p^{t}\right)=0$. Then an arithmetical function $f$ satisfies (8) for all $a \geq b \geq 1$ and $a+b \leq o\left(p^{t}\right)$ if and only if there exists an integer $c$ (depending on $p^{t}$ ) with $1 \leq c \leq o\left(p^{t}\right)$ such that

$$
f\left(p^{i t}\right)=0 \text { for } 1 \leq i \leq c-1 \text { and } 2 c \leq i \leq o\left(p^{t}\right) .
$$

Proof. Since $g\left(p^{t}\right)=0$, then (8) becomes

$$
f\left(p^{(a+b) t}\right) f\left(p^{b t}\right)=0 .
$$

Now, assume that (15) holds. We show that (16) holds. Choose two integers $a, b$ such that $a \geq b \geq 1$ and $a+b \leq o\left(p^{t}\right)$. If $b \leq c-1$, then $f\left(p^{b t}\right)=0$ and consequently (16) holds. If $b \geq c$, then $a+b \geq 2 b \geq 2 c$ and thus $f\left(p^{(a+b) t}\right)=0$. Therefore (16) holds. So we have proved that (16) or (8) holds.

Conversely, suppose that (8) holds, that is, (16) holds. We show that (15) holds. If $f\left(p^{i t}\right)=0$ whenever $1 \leq i \leq o\left(p^{t}\right)$, then (15) holds with $c=1$. Assume then that $f\left(p^{i t}\right) \neq 0$ for some $1 \leq i \leq o\left(p^{t}\right)$. Let $c$ be the smallest $i$ for which $f\left(p^{i t}\right) \neq 0$ and $1 \leq i \leq o\left(p^{t}\right)$. Then $f\left(p^{c t}\right) \neq 0$ and $f\left(p^{i t}\right)=0$ for $i \leq c-1$. Next, suppose $2 c \leq i \leq o\left(p^{t}\right)$. Write $i=a+c(a \geq c)$. Taking $b=c$ in $(16)$ proves that $f\left(p^{i t}\right)=f\left(p^{(a+b) t}\right)=0$. So we have proved that (15) holds. This completes the proof. 
Example 2. Let $g$ be the $A$-multiplicative function with $g\left(p^{t}\right)=0$ for all $A$-primitive prime powers $p^{t}(\neq 1)$. Then $g=\delta$ and (6) becomes

$$
f(m n) f((m, n))=f(m) f(n) \delta((m, n))
$$

for $m, n \in A(m n)$, which means that $f(m n) f(1)=f(m) f(n)$ when $(m, n)=1$ and $f(m n) f((m, n))=0$ when $(m, n)>1$ and $m, n \in A(m n)$. Now, let $c=1$ for all $A$-primitive prime powers $p^{t}(\neq 1)$ in Theorem 3 . Then $f\left(p^{i t}\right)=0$ for $2 \leq i \leq o\left(p^{t}\right)$, which means that $f=\mu_{A} h$ for some quasimultiplicative function $h$. Thus, the function $f=\mu_{A} h$ possesses the property (17). For instance, the function $f=\mu_{A}$ possesses the property (17).

Theorem 4. Let $p^{t}(\neq 1)$ be an A-primitive prime power, and let $g$ be an A-multiplicative function with $g\left(p^{t}\right) \neq 0$. Then an arithmetical function $f$ satisfies (8) if and only if there exists an arithmetical function $h$ (which may depend on $p^{t}$ ) such that

$$
f\left(p^{a t}\right)=h(a) g\left(p^{t}\right)^{a} \text { for all } 1 \leq a \leq o\left(p^{t}\right),
$$

where $h$ satisfies the functional equation

$$
h(a+b) h(b)=h(a) h(b) \text { for all } a \geq b \geq 1, a+b \leq o\left(p^{t}\right) .
$$

Proof. Assume that there exists an arithmetical function $h$ satisfying (19), and let $f\left(p^{a t}\right)$ be given by (18). Then, for $a \geq b \geq 1, a+b \leq o\left(p^{t}\right)$ we have

$$
f\left(p^{(a+b) t}\right) f\left(p^{b t}\right)=h(a+b) g\left(p^{t}\right)^{a+b} h(b) g\left(p^{t}\right)^{b}
$$

and

$$
f\left(p^{a t}\right) f\left(p^{b t}\right) g\left(p^{b t}\right)=h(a) g\left(p^{t}\right)^{a} h(b) g\left(p^{t}\right)^{b} g\left(p^{b t}\right) .
$$

Since $g$ is $A$-multiplicative, $g\left(p^{b t}\right)=g\left(p^{t}\right)^{b}$. Now, by (19), we obtain (8).

Conversely, suppose that (8) holds. Take $h(a)=f\left(p^{a t}\right) / g\left(p^{t}\right)^{a}$ for $1 \leq$ $a \leq o\left(p^{t}\right)$. Then, $f\left(p^{a t}\right)=h(a) g\left(p^{t}\right)^{a}$, and thus (8) becomes

$$
h(a+b) g\left(p^{t}\right)^{a+b} h(b) g\left(p^{t}\right)^{b}=h(a) g\left(p^{t}\right)^{a} h(b) g\left(p^{t}\right)^{b} g\left(p^{b t}\right) .
$$

Since $g\left(p^{b t}\right)=g\left(p^{t}\right)^{b}$ and $g\left(p^{t}\right) \neq 0$, we obtain (19). This completes the proof.

Example 3. Suppose that $f$ is a quasi- $A$-totient and $p^{t}(\neq 1)$ is an $A$ primitive prime power. Then, by Lemma 1 ,

$$
f\left(p^{a t}\right)=f\left(p^{t}\right)\left(\frac{f_{T}\left(p^{t}\right)}{f_{T}(1)}\right)^{a-1}, 1 \leq a \leq o\left(p^{t}\right) .
$$


If $f_{T}\left(p^{t}\right) \neq 0$, then

$$
f\left(p^{a t}\right)=\frac{f_{T}(1) f\left(p^{t}\right)}{f_{T}\left(p^{t}\right)}\left(\frac{f_{T}\left(p^{t}\right)}{f_{T}(1)}\right)^{a}=h(a) g\left(p^{t}\right)^{a},
$$

where $h(a)=f_{T}(1) f\left(p^{t}\right) / f_{T}\left(p^{t}\right)$ (a constant) for all $1 \leq a \leq o\left(p^{t}\right)$ and $g\left(p^{t}\right)=f_{T}\left(p^{t}\right) / f_{T}(1)$. It is clear that $h$ satisfies (19) and $f_{T} / f_{T}(1)$ is an $A$-multiplicative function. Thus $f$ satisfies (8).

\section{References}

[1] Anderson, D.R.; Apostol, T. M.: The evaluation of Ramanujan's sum and generalizations. Duke Math. J. 20 (1953), 211-216.

[2] Apostol, T. M.: Introduction to Analytic Number Theory. SpringerVerlag, 1976.

[3] Apostol, T.M.; Zuckerman, H.S.: On the functional equation $F(m n) F((m, n))=F(m) F(n) f((m, n))$. Pacific J. Math. 14 (1964), 377384.

[4] Chidambaraswamy, J.: On the functional equation $F(m n) F((m, n))=$ $F(m) F(n) f((m, n))$. Portugal. Math. 26 (1967), 101-107.

[5] Cohen, E.: Arithmetical inversion formulas. Canad. J. Math. 12 (1960), 399-409.

[6] Comment, P.: Sur l'equation fonctionnelle $F(m n) F((m, n))=$ $F(m) F(n) f((m, n))$. Bull. Res. Counc. of Israel 7F (1957), 14-20.

[7] Haukkanen, P.: Classical arithmetical identities involving a generalization of Ramanujan's sum. Ann. Acad. Sci. Fenn. Ser. A. I. Math. Dissertationes 68 (1988), 1-69.

[8] Haukkanen, P.: Some characterizations of totients. Internat. J. Math. Math. Sci. 19.2 (1996), 209-218.

[9] Lahiri, D. B.: Hypo-multiplicative number-theoretic functions. Aequationes Math. 9 (1973), 184-192.

[10] McCarthy, P. J.: Introduction to Arithmetical Functions. SpringerVerlag, 1986. 
[11] Narkiewicz, W.: On a class of arithmetical convolutions. Colloq. Math. 10 (1963), 81-94.

[12] Sándor, J.; Crstici, B.: Handbook of Number Theory II. Kluwer Academic, 2004.

[13] Shockley, J. E.: On the functional equation $F(m n) F((m, n))=$ $F(m) F(n) f((m, n))$. Pacific J. Math. 18 (1966), 185-189.

[14] Sita Ramaiah, V.: Arithmetical sums in regular convolutions. J. Reine Angew. Math. 303/304 (1978), 265-283. 283.

[15] Yocom, K. L.: Totally multiplicative functions in regular convolution rings. Canad. Math. Bull. 16 (1973), 119-128. 